\title{
ANÁLISE DA ALTURA DO SORO NO FLUXO DE SAÍDA DE EQUIPO USADO PARA ARTROSCOPIA
}

\author{
ANALYSIS OF THE HEIGHT OF THE SALINE OUTFLOW IN SET USED FOR \\ ARTHROSCOPY
}

Isabel Ziesemer $\operatorname{Costa}^{1}$, Carmem Australia Paredes Marcondes Ribas ${ }^{2}$, Mário Massatomo Namba ${ }^{3}$, Luiz Antonio de Ridder Bauer ${ }^{4}$, Ana Paula Gebert de Oliveira Franco ${ }^{5}$, Edmar Stieven Filho ${ }^{6}$.

\section{RESUMO}

Objetivo: Este trabalho tem como objetivo analisar a influência da altura das bolsas de soro no fluxo de saída, ou vazão, do fluido através de um equipo quatro vias acoplado a uma camisa e ótica artroscópica. Métodos: Foi analisado o tempo de escoamento do soro fisiológico $0,9 \%$ por um equipo quatro vias, acoplado em uma camisa e ótica artroscópica, para atingir os volumes de 2000 $\mathrm{mL}$ e $4000 \mathrm{~mL}$. Foram realizados 20 testes experimentais, divididos em dois grupos: Grupo 1- suporte elevado a uma altura de $80 \mathrm{~cm}$ Grupo 2- suporte elevado a uma altura de $150 \mathrm{~cm}$. Resultados: O fluxo médio nos testes realizados com as bolsas de soro a $80 \mathrm{~cm}$ foi de $7,78 \mathrm{~cm} 3 / \mathrm{s}$ no período de tempo decorrido até o escoamento de $2000 \mathrm{~mL}$ e $6,82 \mathrm{~cm} 3 / \mathrm{s}$ para $4000 \mathrm{~mL}$. Com a elevação das bolsas de soro até $150 \mathrm{~cm}$ o fluxo médio foi para $12,11 \mathrm{~cm} 3 / \mathrm{s}$ durante o período de enchimento dos $2000 \mathrm{~mL}$ e $10,59 \mathrm{~cm} 3 / \mathrm{s} \mathrm{para} 4000 \mathrm{~mL}$. Conclusão: $O$ aumento da altura das bolsas de soro de $80 \mathrm{~cm}$ para $150 \mathrm{~cm}$ gerou um aumento de $60 \%$ na vazão do soro fisiológico.

Palavras-Chaves: Artroscopia, Velocidade de Fluxo, Distenção do Joelho, Visualização

\section{ABSTRACT}

Introduction: This study aims to analyze the influence of the height of the saline bags in output stream or flow of fluid through a 4-spike gravity tubing set delivery unit coupled to a arthroscopic sheath and $4 \mathrm{~mm}$ arthroscope. Methods: Was analyzed the flow time of $0.9 \%$ saline by 4 -spike gravity tubing set, engaged in a arthroscopic sheath and arthroscope, to achieve the volume of 2000 and $4000 \mathrm{~mL}$. Were performed 20 experimental tests, divided in two groups: Group 1 - support raised to a height of $80 \mathrm{~cm}$, Group 2 - support raised to a height of $150 \mathrm{~cm}$. Results: The mean flow on tests with saline bags raised to $80 \mathrm{~cm}$ was 7,78 cm3/s in the time period elapsed until the flow of $2000 \mathrm{~mL}$ and $6.82 \mathrm{~cm} 3 / \mathrm{s}$ to $4000 \mathrm{~mL}$. With the elevation of saline bags to $150 \mathrm{~cm}$, the average flux was $12.11 \mathrm{~cm} 3 / \mathrm{s}$ during the filling of $2000 \mathrm{~mL}$ and $10.59 \mathrm{~cm} 3 / \mathrm{s}$ to $4000 \mathrm{~mL}$. Conclusion: The increases height of the saline bags of $80 \mathrm{~cm}$ to $150 \mathrm{~cm}$ increased to $60 \%$ in the flow of saline solution.

Keywords: Arthroscopy, Flow Velocity, Knee Distention, Visualization.

1. Graduanda em Medicina pela Faculdade Evangélica do Paraná.

2. Doutora em Princípios da Cirurgia pela Faculdade Evangélica do Paraná - FEPAR. Professora adjunta da disciplina de Pediatria da FEPAR.

3. Mestre em Medicina (Clínica Cirúrgica) pela Universidade Federal do Paraná - UFPR. Médico Ortopedista do Ctea - Centro de Traumatologia Esportiva e Artroscopia.

4. Especialista em Ortopedia e Traumatologia pelo Hospital Universitário Cajuru. Médico Ortopedista do Ctea Centro de Traumatologia Esportiva e Artroscopia.

5. Doutora em Odontologia pela Pontifícia Universidade Católica do Paraná - PUCPR. Coordenadora de Pesquisa Científica do Ctea - Centro de Traumatologia Esportiva e Artroscopia.

6. Doutorando em Medicina (Clínica Cirúrgica) pela Universidade Federal do Paraná - UFPR. Médico Ortopedista do Ctea - Centro de Traumatologia Esportiva e Artroscopia. 


\section{INTRODUÇÃO}

Uma visualização adequada é imperativa para a realização de um procedimento artroscópico.(1) 0 líquido sinovial não é um bom meio de visualização, por isso é substituído por gás ou solução salina na artroscopia.(2) Durante o procedimento um volume contínuo de fluido é necessário para manter a distensão articular. Essa distensão determina o campo cirúrgico intra-articular.(3) A pressão intra-articular e o fluxo têm influência significativa na visualização adequada do campo artroscópico.(4) A correta visualização do campo cirúrgico determina maior segurança e rapidez do procedimento artroscópico. $(5,6)$

Existem diferentes sistemas de irrigação para a manutenção do fluxo da solução salina intra-articular. Eles podem ser baseados na gravidade, automatizados e pneumáticos. Os gravitacionais são de baixo custo, porém podem apresentar problemas como a perda de pressão intra-articular. Essa perda induz à hemorragia da articulação, piora da visualização e interrupção do ato cirúrgico.(7)

Quando o sistema de irrigação é dependente da gravidade, o fluxo é alterado pela altura da bolsa de soro em relação ao nível de saída do líquido.(2) Uma forma de manter a pressão seria elevar as bolsas de soro, aumentando assim a altura da coluna líquida.

Este trabalho tem como objetivo analisar a influência da altura das bolsas de soro no fluxo de saída, ou vazão, do fluido através de um equipo quatro vias acoplado a uma camisa e ótica artroscópica.

\section{METODOLOGIA}

Para elevar as bolsas de soro fisiológico, um suporte metálico telescópico, com sistema de roldanas foi desenvolvido pelos autores. O suporte tem quatro pontos de apoio para colocar os soros. O suporte atinge uma altura máxima de cinco metros em relação ao chão (Figura 1).

Foram realizados 20 testes experimentais, os quais foram divididos em dois grupos de acordo com a altura do suporte das bolsas de soro fisiológico $0,9 \%$. Grupo 1- suporte elevado a uma altura de $80 \mathrm{~cm}(\mathrm{~N}=$ 10), Grupo 2- suporte elevado a uma altura de $150 \mathrm{~cm}$ $(N=10)$. As alturas foram determinadas em relação ao orifício de saída do equipo quatro vias. Quatro bolsas de soro fisiológico foram utilizadas para cada teste.

A coleta de dados foi realizada no Laboratório de Pesquisa do Centro de Traumatologia Esportiva e Artroscopia - Ctea ${ }^{\circledR}$, com a utilização dos seguintes materiais: 80 bolsas de soro fisiológico de $1000 \mathrm{~mL}$ da marca $\mathrm{JP}^{\circledR}$, um equipo quatro vias (Zammi Instrumental Ltda $^{\circledR}$, Duque de Caxias, RJ, Brasil), camisa artroscópica de alto fluxo KARL STORZ ${ }^{\circledR} 28229$ CR High Flow Sheath, ótica artroscópica KARL STORZ ${ }^{\circledR} 3,5 \mathrm{~mm}$, copo de Becker de $10.000 \mathrm{~mL}$ e filmadora SONY ${ }^{\circledR}$ DCR-SX45 para a gravação dos testes (Figura 2).

As filmagens, feitas pela câmera, foram utilizadas para mensurar o tempo de escoamento do soro fisiológico no copo Becker. Posteriormente, os vídeos foram analisados por meio do software de edição de vídeo Camtasia 7.1 (C2010 TechSmith.

O tempo de escoamento do soro fisiológico foi contabilizado a partir do momento da abertura da válvula da ótica artroscópica e registrado em dois momentos, quando o nível do fluido no copo de Becker atingiu $2000 \mathrm{~mL}$ e $4000 \mathrm{~mL}$.

Os dados obtidos foram analisados estatisticamente no Programa Statistical Package for the Social Sciences - SPSS (Statistical Products and Service Solutions, Chicago, Illinois, USA) por meio do teste-T para duas amostras presumindo variâncias equivalentes, considerando $p<0,05$.

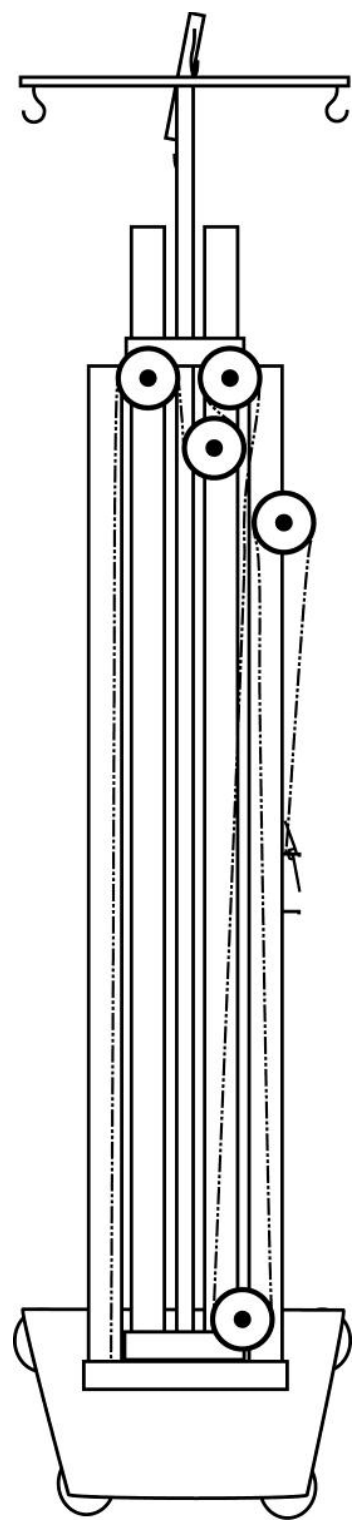

Figura 1- Suporte 


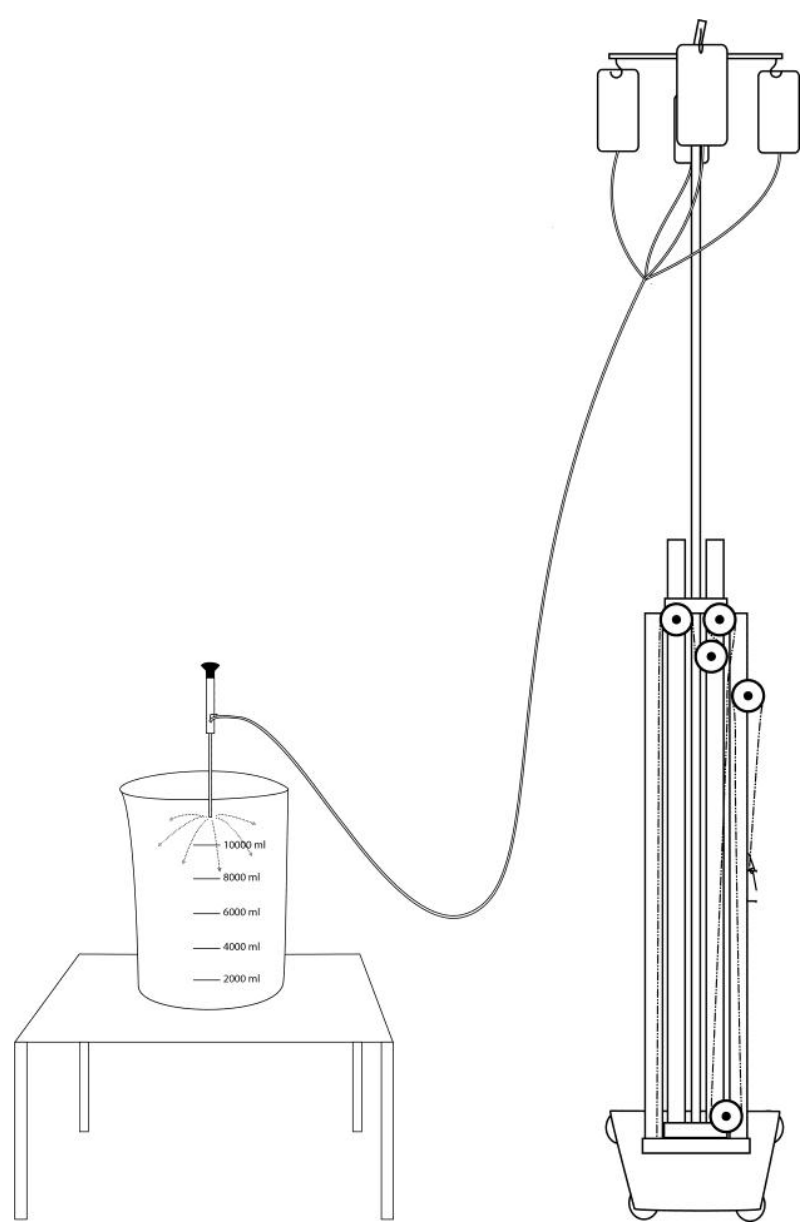

Figura 2

\section{RESULTADOS}

Os resultados dos testes estão descritos na Tabela 1.

O fluxo médio nos testes realizados com as bolsas de soro a $80 \mathrm{~cm}$ foi de $7,78 \mathrm{~cm} 3 / \mathrm{s}$ no período de tempo decorrido até o escoamento de $2000 \mathrm{~mL}$. No entanto houve uma diminuição do fluxo médio para $6,82 \mathrm{~cm} 3 / \mathrm{s}$ quando considerado o tempo total para atingir o volume de $4000 \mathrm{~mL}$ de soro dentro do copo de Becker. Com a elevação das bolsas de soro até $150 \mathrm{~cm}$ o fluxo médio foi para $12,11 \mathrm{~cm} 3 / \mathrm{s}$ durante o período de enchimento dos $2000 \mathrm{~mL}$, com uma redução para $10,59 \mathrm{~cm} 3 / \mathrm{s}$ quando considerado o tempo total para $4000 \mathrm{~mL}$.

A elevação das bolsas de soro fisiológico resultou em uma diminuição significativa nos tempos de escoamento do soro até atingir o volume de 2000 $\mathrm{mL}$ e também para $4000 \mathrm{~mL}$, com $\mathrm{p}<0,0001$ em ambos. Este resultado significa um aumento real da velocidade de saída do soro de aproximadamente $60 \%$ com o incremento da altura tanto para atingir os $2000 \mathrm{~mL}$ de volume como $4000 \mathrm{~mL}$.
Tabela 1. Tempo em segundos decorridos para escoar $2000 \mathrm{~mL}$ e $4000 \mathrm{~mL}$ com as bolsas de soro nas alturas de $80 \mathrm{~cm}$ e $150 \mathrm{~cm}$.

\begin{tabular}{lll}
\hline Volume (mL) & 2000 & 4000 \\
\hline Tempo (s) média & 257 & 586,5 \\
\hline Desvio Padrão & $\pm 5,72$ & $\pm 10,16$ \\
Tempo (s) média 2 & 165,1 & 377,6 \\
Desvio Padrão2 & $\pm 3,81$ & $\pm 3,13$ \\
\hline
\end{tabular}

\section{DISCUSSÃO}

Três sistemas de irrigação são comumente utilizados: sistemas de bombeamento mecânico automatizados, bolsas salinas pressurizadas e sistemas dependentes da gravidade.(1)

Nos sistemas de bombeamento mecânico o objetivo principal é manter a articulação distendida continuamente acima da pressão crítica de fechamento das arteríolas, mantendo uma hemostasia efetiva.(2) A bomba de infusão proporciona uma melhora da visualização do campo cirúrgico, porém a sua utilização é limitada principalmente pelo alto custo que ela apresenta. A maior parte das operadoras de saúde presentes no território nacional não reconhecem a necessidade este equipamento.(7)

Como desvantagem, nesses sistemas a pressão deve ser controlada para que não atinja gradientes pressóricos excessivos. Pressões intraarticulares muito elevadas podem romper a capsula ou até causar síndrome do compartimento e comprometimentos vasculares nos extravasamentos para o espaço poplíteo e região do canal femoral.(3,813)

As bolsas pressurizadas são jaquetas infláveis colocadas ao redor das bolsas de soro, que são manualmente infladas à medida que é necessário aumentam a pressão intra-articular.(4)

Os sistemas dependentes da gravidade são baratos, seguros porém com efetividade menor que os mecânicos.(7)

Fluxo, velocidade e pressão são conceitos importantes para entender o processo de visualização em artroscopia, principalmente quando se usa os sistemas de gravidade. O fluxo é determinado pela razão entre o volume de fluido escoado e o tempo decorrido, e é dado em $\mathrm{cm} 3 / \mathrm{s}$. O fluxo através de um tubo segue a lei de Poiseuille. Pode-se dizer que este tipo de fluxo é a razão do gradiente de pressão e do diâmetro da secção perpendicular do tubo elevado à 
quarta potência, pelo comprimento do tubo e viscosidade do fluido.(14) Ou seja, quanto mais alto e maior o orifício de saída, maior o fluxo. Quanto mais viscoso o líquido e mais comprido o equipo, menor.

O fluxo é importante pois é ele que limpa os detritos da articulação durante um procedimento. Mesmo com alta pressão articular, capaz de bloquear o sangramento arteriolar, se não houver um bom fluxo a articulação pode ficar com a visualização difícil.

O fator mais importante para a regulação do fluxo é o diâmetro do orifício de saída do soro.(14) Como o aumento do diâmetro do tubo tem influência na vazão, o uso de camisa de alto fluxo pode ser uma opção importante na escolha do material. Materiais que apresentam pequenos orifícios para saída do soro podem causar déficit de visualização e prejuízo no desempenho cirúrgico.

O uso de cânula exclusiva para infusão pode resolver o problema de fluxo em caso de camisa artroscópica com pequenos orifícios. Uma desvantagem deste sistema é que o fluxo não é direcionado diretamente para a área de interesse primário, a área visualizada pela ótica.(2)

O aumento do fluxo também pode ser conseguido com a diminuição do comprimento do tubo. Porém em procedimentos cirúrgicos essa ação fica limitada, pois é preciso um comprimento mínimo do equipo quatro vias para que o cirurgião tenha liberdade de movimento.

A pressão que um fluido exerce em um determinado ponto é determinada pela Lei de Stevin, onde se multiplica a altura da coluna líquida, a aceleração da gravidade e a densidade do fluido.(14) Ou seja, quanto mais alto, maior a força da gravidade e mais denso o líquido, maior a pressão.

Dos itens que influenciam na pressão, a altura é a única variável que pode ser alterada facilmente em procedimento cirúrgico com sistemas de irrigação por gravidade. No caso deste trabalho a vazão a vazão aumentou em $60 \%$ com a elevação em $70 \mathrm{~cm}$ das bolsas de soro. Isso ocorreu pois a elevação levou a um aumento da pressão do fluido no orifício de saída, consequentemente aumentando sua vazão.(2)

A pressão intra-articular mínima adequada para a realização da artroscopia é de $55 \mathrm{mmHg}$. Ela é atingida com uma coluna de soro fisiológico a 0,9\% de $75 \mathrm{~cm}$. Essa pressão é importante para promover a homeostase arteriolar, diminuindo ou impedindo o sangramento durante um procedimento artroscópico.(1)

Uma das desvantagens dos sistemas gravitacionais e das bolsas pressurizadas é que à medida que as bolsas de soro esvaziam há a perda do fluxo adequado de soro e uma diminuição da pressão intra-articular.(4) A perda de pressão do fluido pode causar hemorragia da articulação, perda da visualização e interrupção do ato cirúrgico.(7)
Outra desvantagem referente ao sistema gravitacional é a necessidade da utilização de grandes volumes de fluido. Apesar dessas desvantagens, a cirurgia artroscópica pode ser realizada com o sistema gravitacional não pressurizado.(1,3)

O material cirúrgico é fundamental para o bom andamento do procedimento. Camisas de alto fluxo, sistemas mecânicos de infusão e adição de cânula de infusão ajudam na visualização do campo artroscópico. Associado a isto, nos casos em que a infusão dependente de gravidade, seguindo-se os devidos cuidados, um suporte de sala mais alto e que permita uma troca de soro mais rápida pode ser importante para o desempenho cirúrgico.

\section{CONCLUSÃO}

O aumento da altura das bolsas de soro de 80 $\mathrm{cm}$ de para $150 \mathrm{~cm}$ gerou um aumento $60 \%$ na vazão do soro fisiológico através do equipo quatro vias acoplado a uma camisa e ótica artroscópica.

\section{REFERÊNCIAS}

1. Arangio G, Kostelnik KE. Intraarticular pressures in a gravity-fed arthroscopy fluid delivery system. Arthroscopy 1992;8(3):341-4.

2. Oretorp N, Elmersson S. Arthroscopy and irrigation control. Arthroscopy 1986;2(1):46-50.

3. Noyes FR, Spievack ES. Extraarticular fluid dissection in tissues during arthroscopy. A report of clinical cases and a study of intraarticular and thigh pressures in cadavers. The Am J Sports Med 1982;10(6):346-51.

4. Ramoutar D, Titchener A, Gormley C, Becker G. A reliable method of maintaining constant arthroscopy irrigation pressure. Injury 2010;41(7):772-3.

5. Morgan CD. Fluid delivery systems for arthroscopy. Arthroscopy 1987;3(4):288-91.

6.Mueller T, Menth-Chiari W. Accuracy of pressure and flow capacities of four arthroscopic fluid management systems. Arthoscopy 2001;7(7):760-4.

7.Davison J, Strover A. A technique for prevention of sudden pressure loss on emptying of irrigation bags during arthroscopic surgery using gravity-fed irrigation systems. Arthroscopy 1993;9(3):336-7.

8. Abbushi W, Egbert R, Pichelmeier R, Kovacs J. Compartment syndrome of the forearm due to infusion and transfusion using a pressure pump. Anasthesiol Intensivmed Notfallmed Schmerzther: AINS 1991;26(6):348-51.

9. Burgaard P, Blyme PJ, Olsen PM, Kristensen G. Rupture of the knee capsule from articular hyperpressure. Experiments in cadaver knees. Acta Orthop Scand 1988;59(6):692-4.

10.Carpenter JF. Complications associated with the use of an infusion pump during knee arthroscopy. Arthroscopy 1993;9(2):244.

11.Ekman EF, Poehling GG. An experimental assessment of the risk of compartment syndrome during knee arthroscopy. Arthroscopy 1996;12(2):193-9.

12.Funk D a, Noyes FR, Grood ES, Hoffman SD. Effect of flexion angle on the pressure-volume of the human knee. Arthroscopy 1991;7(1):86-90.

13. Romero J, Smit CM, Zanetti M. Massive intraperitoneal and extraperitoneal accumulation of irrigation fluid as a complication during knee arthroscopy. Arthroscopy;14(4):401-4.

14. Okuno E, Caldas IL, Chow C. Física para ciencias biológicas e biomédicas. HARBRA; 1982. 